\title{
Immune Checkpoint Inhibitor Therapy in Breast Cancer
}

\author{
Cesar A. Santa-Maria, MD, MSCI, ${ }^{a}$ and Rita Nanda, MD $^{\mathrm{b}}$
}

\begin{abstract}
Initial studies investigating single-agent activity of immune checkpoint inhibitors (ICls) serve as proof of principle that harnessing the immune system can have anticancer activity in a variety of human malignancies. Although breast cancer was historically believed to be immunogenically silent, early studies indicate overall response rates with ICls are similar to those observed with many other solid malignancies. Overall response rates in advanced breast cancer are low, but the responses are remarkably durable. A deeper understanding of the biology of the interaction between cancer and immune cells is required to both develop biomarkers that more accurately predict response to therapy and identify effective immunotherapy-based combination strategies that can enhance the immunogenicity of biologically "cold" tumors. Breast cancer encompasses a variety of diseases defined by the presence or absence of central oncogenic drivers, and early data suggest that the distinct subtypes may have unique immune phenotypes. Breast cancer represents an ideal disease in which to investigate immunotherapeutic strategies given the prevalence of the disease, unique clinical trial design opportunities, and immunophenotypic diversity.
\end{abstract}

J Natl Compr Canc Netw 2018;16(10):1259-1268 doi: 10.6004/jncen.2018.7046

\begin{abstract}
A hallmark of cancer is the ability to evade the immune system through tumor-mediated immune escape mechanisms. ${ }^{1}$ Immune evasion can occur via a number of mechanisms, including the manipulation of key immune checkpoints that regulate the adaptive immune system, including CTLA-4, PD-1, and PD-L1. CTLA-4 inhibitors were the first immune checkpoint inhibitors (ICIs) to demonstrate a benefit in melanoma; subsequently PD-1/PD-L1 inhibitors have shown response rates across a wide variety of cancers ranging from $10 \%$ to $27 \% .^{2-7}$ Although the FDA has approved a number of ICIs for a variety of cancers, the proportion of patients who benefit from single-agent ICIs is low. However, these monotherapy studies have demonstrated that immunotherapy can elicit durable antitumor responses. Understanding the interaction between the immune system and cancer is crucial for identifying patients most likely to respond to treatment and for optimizing
\end{abstract}

\footnotetext{
aBreast and Ovarian Cancer Program, Johns Hopkins Sidney Kimmel Comprehensive Cancer Center, Baltimore, Maryland, and ${ }^{\mathrm{b}}$ Department of Medicine, Section of Hematology/Oncology, The University of Chicago, Chicago, Illinois.

Submitted December 16, 2017; accepted for publication May 7, 2018.
}

Dr. Santa-Maria has disclosed that he receives grant/research support from immunotherapy-based treatment strategies. This article reviews the experience with immune checkpoint blockade in breast cancer and future directions in therapeutic and biomarker strategies.

\section{Single-Agent Immune Checkpoint Experience}

Although there are many molecular subtypes of breast cancer, clinically they are classified by the presence or lack of endocrine receptors (ERs) and HER2. ${ }^{8,9}$ Response rates to immunotherapy in breast cancer vary among the different clinical subtypes of breast cancer. Breast cancer subtypes are both genetically and phenotypically distinct, and although this may not be the optimal classification to assess immunotherapy sensitivity, they provide a unique consideration regarding drug development strategies. Early studies suggest that breast cancer lacking these major receptors-triple-negative

Pfizer and Medimmune, and serves on the advisory board for Polyphor. Dr Nanda has disclosed that she serves on the advisory board for AstraZeneca, Celgene, Genentech, Merck, Pfizer, Puma, and Syndax.

Correspondence: Cesar A. Santa-Maria, MD, MSCI, Breast and Ovarian Cancer Program, The Sidney Kimmel Comprehensive Cancer Center at Johns Hopkins, 201 North Broadway, Suite 10262, Baltimore, MD 21231. Email: csantam2@jhmi.edu 
breast cancer (TNBC) - appears to be the most immunosensitive of the subtypes.

\section{Triple-Negative Breast Cancer}

To date, TNBC is the breast cancer subtype in which immune checkpoint blockade has shown the most promise. The KEYNOTE-012 study was a multicohort phase IB study that evaluated the safety and tolerability of the PD-1 inhibitor pembrolizumab in multiple tumor types, including metastatic TNBC. Patients were eligible for enrollment if their tumors were positive for PD-L1 expression (at least $1 \%$ of tumor or infiltrating immune cells were positive for PDL1 by immunohistochemistry). The overall response rate (ORR) in the 32-patient cohort was 18.5\% (95\% CI, $6.3 \%-38.1 \%)$, median time to response was 17.9 weeks (range, 7.3-32.4 weeks), and median duration of response was not reached ( 15 to $\geq 47.3$ weeks). ${ }^{10}$ The subsequent phase II KEYNOTE-086 study evaluated the efficacy of pembrolizumab in a larger cohort of patients with advanced TNBC. Cohort A included patients with previously treated metastatic TNBC (including patients with both PD-L1-positive and -negative disease); cohort $\mathrm{B}$ included patients with previously untreated metastatic PD-L1-positive TNBC. ${ }^{11,12}$ ORRs in cohorts A and B were $4.7 \%$ and $23 \%$, respectively. Lactate dehydrogenase (LDH) levels and the percentage of patients with visceral metastases were slightly higher in cohort A than B (51\% vs $40 \%$ and $74 \%$ vs $69 \%$, respectively). Median time to response was a month longer in cohort $\mathrm{A}$ (12 weeks) than in cohort B (8.7 weeks). Notably, the ORR in cohort A was similar irrespective of PD-L1 status; all patients in cohort $\mathrm{B}$ were required to have PD-L1positive disease. These findings suggest that certain clinical features, such as line of therapy and disease burden, correlate with response and may be important patient selection criteria.

Another study investigating the PD-L1 inhibitor atezolizumab in metastatic TNBC demonstrated an ORR of $10 \%$ in 112 evaluable patients unselected for PD-L1 status. ${ }^{13}$ In this study, PD-L1 positivity was defined as $>5 \%$ staining of tumor-infiltrating immune cells on immunohistochemistry, and patients who were PD-L1-positive had longer median overall survival (OS) compared with those who were PD-L1-negative (10.7 months [95\% CI, 7.2-14.7] vs 7.1 months [95\% CI, 5.1-12.6], respectively). However, higher levels of tumor-infiltrating lymphocytes (TILs, $>10 \%$ ) versus lower levels was a more discriminating biomarker of median OS (12.6 months [95\% CI, 10.5-not reached) vs 6.6 months [95\% CI, 4.9-10.2], respectively). Finally, as in the KEYNOTE-086 study, line of therapy was an important determinant of response, with an ORR of $26 \%$ in patients who had not received any therapy for advanced-stage disease, whereas those who had received at least one line of therapy for metastatic breast cancer had an ORR of $<8 \%$. The phase IB JAVELIN study investigated avelumab, an anti-PDL1 antibody, in various breast cancer subtypes. Avelumab monotherapy was associated with an ORR of $5.2 \%$ in the TNBC subgroup $(n=58)$, which increased to $22.2 \%$ in PD-L1-positive TNBC (defined as PD-L1 expression >10\%). ${ }^{14}$

\section{Hormone Receptor-Positive Breast Cancer}

Although preliminary data suggest response rates may be lower in ER-positive disease compared with TNBC, durable responses have been observed with immune checkpoint blockade. A clinical trial of 26 patients with estrogen receptor (ER)-positive metastatic breast cancer treated with the CTLA-4 inhibitors tremelimumab and exemestane found an increase in peripheral CD4-positive and CD8-positive $\mathrm{T}$ cells relative to FOXP3-positive regulatory $\mathrm{T}$ cells, which may be suggestive of a robust antitumor immune response. Furthermore, $42 \%$ of patients experienced stable disease (SD) for at least 3 months despite previously progressing on antihormone therapy, although no benefit was observed among patients with prior exemestane $(n=5) \cdot{ }^{14,15}$ In the aforementioned JAVELIN study that included 72 patients with ER-positive, HER2-negative breast cancer, 2 responses were observed (ORR, 2.8\%), although only $55 \%$ of patients had PD-L1 expression $\geq 1 \%{ }^{16}$ The KEYNOTE-028 study with pembrolizumab reported an ORR of $12 \%$ for patients with ER-positive disease expressing PD-L1, although this was a heavily pretreated population. ${ }^{17}$

\section{HER2-Positive Breast Cancer}

HER2-positive breast cancer can be associated with a robust immune infiltrate, suggesting a role for immunotherapy in this breast cancer subtype. Initial studies have demonstrated a relatively low response rate to checkpoint monotherapy in a heavily pretreated HER2-positive population. The only presented data 
Immune Checkpoint Inhibitors in Breast Cancer

to date in HER2-positive breast cancer are from the JAVELIN study, which included 26 patients with heavily pretreated HER2-positive disease. ${ }^{14}$ Among these patients, there were no confirmed responses. Although monotherapy response rates were not observed in the JAVELIN study, combining ICIs with HER2-directed therapy may be more beneficial. Trastuzumab, a monoclonal antibody against HER2, primarily works through antibody-dependent cellular cytotoxicity, which may benefit from immune-stimulating therapies. The PANACEA study investigated pembrolizumab in combination with trastuzumab in HER2-positive metastatic breast cancer, and the ORR in patients with PD-L1-positive disease was found to be $15 \%$ (95\% CI, $7 \%-27 \%) .{ }^{18}$ Of note, patients had previously received and progressed on trastuzumab; therefore, responses were likely due primarily to pembrolizumab rather than demonstrating a true synergistic response with trastuzumab. Responses were not observed in patients with PD-L1-negative disease. PD-L1 status strongly correlated with baseline stromal TIL infiltration $(P=.0004)$, and patients with $\geq 5 \%$ stromal TILs versus $<5 \%$ had an ORR of $39 \%$ versus $5 \%$, respectively. A phase II study is now investigating TDMI with or without atezolizumab (ClinicalTrials. gov identifier: NCT02924883).

\section{Combination Approaches Using Immunotherapy}

Next-generation studies are now investigating how to combine immune checkpoint blockade with novel agents that can potentially "prime" tumors that are not immunogenic (ie, immune cold) phenotypes into ones that are (immune hot) in order to augment response rates. Table 1 summarizes reported combinations in metastatic breast cancer. These approaches are now being investigated in the early-stage setting, where the implications may include improving cure rates.

\section{Chemotherapy Combinations}

There is an intricate, bidirectional relationship between chemotherapy and the immune system. Chemotherapy requires an intact immune system to have maximal antitumor efficacy, and can modulate the immunogenicity of tumors. The efficacy of numerous chemotherapeutic agents is decreased in immunedeficient states. ${ }^{19}$ Furthermore, conventional che- motherapy can enhance the immunogenic properties of malignant cells, which may stimulate immune effector cells. Chemotherapy can potentially increase antigen release and improve immunogenicity, which may result in increased susceptibility to the immune system. ${ }^{19}$

A phase IB study in metastatic TNBC investigated atezolizumab with nab-paclitaxel, and found the ORR to be $38 \%$. The study did not require tumor PD-L1 positivity, and responses were seen irrespective of PD-L1 status. ${ }^{20}$ These results will be validated in the randomized phase III IMpassion130 study, which randomized patients to nab-paclitaxel \pm atezolizumab (ClinicalTrials.gov identifier: NCT02425891). A press release has declared that the primary end point of progression-free survival was met in the intention-to-treat and PD-L1-positive first-line populations, making this the first phase III study in breast cancer to show a survival benefit to immunotherapy. ${ }^{21}$ In another study investigating pembrolizumab + eribulin in 106 patients with metastatic TNBC, the ORR was $26.4 \%$ (95\% CI, 18.3\%$35.9 \%)$ and response rates were similar regardless of PD-L1 status. ${ }^{22}$ Another small study ( $\left.\mathrm{n}=12\right)$ evaluating pembrolizumab with capecitabine in metastatic TNBC found the ORR to be $42 \% .^{23}$

The KEYNOTE-355 study is a randomized phase III study investigating chemotherapy with or without pembrolizumab in the frontline setting in metastatic TNBC. In an effort to better understand which chemotherapy agent would pair best with immunotherapy, the TONIC trial randomized patients with metastatic TNBC to receive nivolumab with either radiation, doxorubicin, cisplatin, or cyclophosphamide. ${ }^{24}$ The authors observed that the response rate was highest with doxorubicin (ORR, 35\%), followed by cisplatin (ORR, 23\%). Single-agent nivolumab (ORR, 17\%) had a better response rate then either radiation (ORR, 8\%) or cyclophosphamide (ORR, $8 \%)$. It is important to note that this trial included a small number of patients $(n=66)$ randomized into multiple arms, and the study was not powered for comparison between arms.

The most promising evidence of the synergism between chemotherapy and immunotherapy was demonstrated in the I-SPY2 phase II neoadjuvant clinical trial. ${ }^{25}$ This study investigated the addition of pembrolizumab to paclitaxel followed by dose-dense doxorubicin with cyclophosphamide in HER2-nega- 
Santa-Maria and Nanda

\begin{tabular}{|c|c|c|c|c|}
\hline Treatment & Phase & Breast Cancer Subtype (n) & ORR & Reference \\
\hline Atezolizumab + nab-paclitaxel & IB & TNBC (32) & $38 \%$ & Adams et al, ${ }^{20} 2016$ \\
\hline Pembrolizumab + eribulin & $\mathrm{IB} / \mathrm{II}$ & TNBC (106) & $26.4 \%$ & Tolaney et al, 222017 \\
\hline Pembrolizumab + capecitabine & Pilot & TNBC (12) & $42 \%$ & Page et al, ${ }^{23} 2018$ \\
\hline Durvalumab + tremelimumab & Pilot & $\begin{array}{l}\text { All patients (18) } \\
\text { ER-positive (11) } \\
\text { TNBC (7) }\end{array}$ & $\begin{array}{l}17 \% \\
0 \% \\
43 \%\end{array}$ & Santa-Maria et al, ${ }^{29} 2018$ \\
\hline $\begin{array}{l}\text { Tremelimumab + brain radiation } \\
\text { (+ trastuzumab if HER2-positive) }\end{array}$ & Pilot & $\begin{array}{l}\text { All patients (26) } \\
\text { HER2-negative (20) } \\
\text { HER2-positive (6) }\end{array}$ & $\begin{array}{l}4 \% \text { (non-CNS ORR) } \\
0 \% \text { (non-CNS ORR) } \\
17 \% \text { (non-CNS ORR) }\end{array}$ & McArthur et al, ${ }^{42} 2017$ \\
\hline Pembrolizumab + radiation & Pilot & TNBC (9) & $33 \%$ & McArthur et al, ${ }^{43} 2018$ \\
\hline Durvalumab + olaparib & IB & $\begin{array}{l}\text { All patients (12) } \\
\text { Ovarian (10) } \\
\text { TNBC (2) }\end{array}$ & $\begin{array}{l}17 \% \\
20 \% \\
0 \%\end{array}$ & Lee et $a l,{ }^{53} 2017$ \\
\hline Pembrolizumab + niraparib & II & TNBC (46) & $28 \%$ & Vinayak et al, ${ }^{54} 2018$ \\
\hline
\end{tabular}

Abbreviations: CNS, central nervous system; ER, estrogen receptor; ORR, overall response rate; TNBC, triple-negative breast cancer.

tive early-stage breast cancer. In the TNBC cohort, the Bayesian model estimated that the pathologic complete response (pCR) rate was 60\% with the addition of pembrolizumab versus $20 \%$ without-a tripling of the estimated $\mathrm{pCR}$ rate. Likewise in the ER-positive cohort, the estimated pCR rate nearly tripled, from $13 \%$ to $34 \%$, with the addition of pembrolizumab-an unprecedented improvement in the estimated $\mathrm{pCR}$ rate in this breast cancer subtype. The estimated $\mathrm{pCR}$ rate seen for the TNBC cohort was lower than what has been reported for anthracycline and taxane regimens in other studies. ${ }^{26} \mathrm{~A}$ number of patients in the control arm received carboplatin in addition to chemotherapy if they were found to have a suboptimal response to standard chemotherapy. Even if these patients went on to achieve a pCR, for the purposes of the study, they were counted as non-pCR because they deviated from their assigned treatment. Although most toxicities were consistent with what has been observed with taxane- and anthracycline-based therapy, there was a disproportionately higher incidence of immune-related toxicities, the most common being thyroid function abnormalities (hyperthyroidism and hypothyroidism) and adrenal insufficiency (both primary and secondary). Adrenal insufficiency was observed in 6 of 69 patients randomized to pembrolizumab $(8.7 \%$ ) - a reminder of the potential toxicity of ICIs and the importance of long-term follow-up as these agents are translated into the early-stage, curative setting.

These data can be contrasted with those of the recently reported GeparNuevo study, which random- ized 174 patients with early-stage TNBC to receive nab-paclitaxel followed by epirubicin and cyclophosphamide \pm durvalumab. ${ }^{26}$ Investigators found a pCR rate of $53 \%$ in the durvalumab arm and $44 \%$ in the control arm; however, these were not statistically different $(P=.287$; adjusted odds ratio, $1.53 ; 95 \% \mathrm{CI}$, $0.82-2.84 ; P=.182)$. The study was designed to give some patients $(n=177)$ a 2 -week "window" of durvalumab or placebo before receiving chemotherapy ( \pm continued durvalumab according to their initial treatment allocation). The authors noted that in the "window" cohort, patients in the durvalumab arm had a better pCR than those in the placebo arm $(61 \%$ vs $41 \%$, respectively), suggesting that induction durvalumab may be beneficial. The investigators did note that younger patients (aged $<40$ years) and those with higher stage disease had numerically higher $\mathrm{pCR}$ rates if durvalumab was added versus placebo.

\section{Immune-Based Combinations}

Augmenting the immune response with a variety of other ICIs is an important strategy being investigated. Inhibition of both CTLA-4 and the PD-1/ PD-L1 axis has demonstrated clinical benefit in several tumor types. ${ }^{27,28}$ A pilot study of 18 patients investigating tremelimumab and durvalumab in metastatic breast cancer found this combination to have an ORR of $17 \%$ in an unselected population; however, responses were only observed in TNBC (ORR, 43\%) rather than ER-positive disease (ORR, 0\%). An expansion cohort in TNBC for this study is currently accruing (ClinicalTrials.gov identi- 
fier: NCT02536794). ${ }^{29}$ This trend closely mirrors that seen with single-agent PD-1/PD-L1 inhibitors, wherein TNBC tends to have higher responses to immune-stimulatory therapy than ER-positive disease, but further validation is required.

Indoleamine 2,3-dioxygenase (IDO) is an immune checkpoint that is involved in immune tolerance. It is an enzyme that breaks down tryptophan, which can lead to effector T-cell apoptosis and growth arrest. IDO can be expressed on tumor cells, but also on cells in the microenvironment. In melanoma, the IDO inhibitor epacadostat has been combined with nivolumab in patients with advanced solid tumors; ORR in treatment-naïve melanoma was $63 \%$, although it was $23 \%$ in head and neck cancer. ${ }^{30}$ This drug class is being investigated in multiple other malignancies, including breast cancer (ClinicalTrials.gov identifier: NCT02178722).

Vaccines have been investigated in breast cancer for many years with limited success. Most studies have focused on peptide-based vaccines in the adjuvant setting derived from antigenic epitopes of tumor-associated antigens, such as HER2, MUC-1, cancer testis antigens, human telomerase reverse transcriptase (hTERT), carcinoembryonic antigen (CEA), p53, and mammaglobin-A (MAM-A). ${ }^{31}$ HER2 peptide vaccines targeting the E75 peptide nelipepimut-S in combination with granulocytemacrophage colony-stimulating factor (GM-CSF) have been studied in a phase I/II trial in the adjuvant setting, demonstrating that disease-free survival (DFS) may be improved in the vaccinated versus unvaccinated group (5-year DFS, $89.7 \%$ vs $80.2 \%$, respectively; $P=.008$; 5 -year DFS, $94.6 \%$ in those optimally vaccinated; $P=.05) .{ }^{32}$ Tumors in this study could have any degree of HER2, and these findings have resulted in larger confirmatory studies (ClinicalTrials.gov identifier: NCT01479244). GM-CSFsecreting vaccines have been found to have potent antitumor effects in preclinical models, although as a single agent they have not been found to produce significant clinical benefit. Preclinical studies of GM-CSF-secreting vaccines in combination with immunomodulatory doses of cyclophosphamide and trastuzumab have demonstrated synergistic antitumor effects. This combination was studied in an early-phase study in patients with HER2-positive metastatic breast cancer, showing a clinical benefit rate at 6 months of $55 \%$ (95\% CI, 32\%-77\%; P=.013) and median progression-free survival of 7 months (95\% CI, 4-16 months). ${ }^{33}$ These data have led to a larger randomized phase II study (NCT00971737). With the development of ICIs, the vaccines may offer a personalized approach to immunotherapy, and are poised to have greater success in clinical trials.

\section{Cryotherapy Combinations}

A mechanism of resistance to immunotherapy in breast cancer may be failure of the immune system to recognize the tumor, namely lack of immunogenic neoantigens. ${ }^{34}$ Cryotherapy can cause cell death and initiate inflammation, which can expose intact neoantigens that can be recognized by the immune system. ${ }^{35}$ A pilot study investigating a single dose of preoperative ipilimumab followed by cryotherapy in a largely ER-positive cohort (72\% of patients) found that therapy was associated with sustained elevations in CD8-positive T cells and Th1 cytokines, suggesting an induced synergistic antitumor immunity. ${ }^{36}$ Deep sequencing of T-cell receptor DNA correlated with TIL assessment on hematoxylin-eosin-stained sections, and cryoablation was associated with an expansion of peripheral blood and intratumoral Tcell clones, suggesting that these may be potential biomarkers of T-cell responses. ${ }^{37}$ These results demonstrate favorable immunomodulation and have led to additional studies now investigating cryotherapy with ipilimumab and nivolumab (ClinicalTrials.gov identifier: NCT02833233).

\section{Radiation Combination}

Radiation can promote release of inflammatory chemokines in the microenvironment as well as neoantigen release, which leads to recruitment of antigenpresenting cells and activated cytotoxic $\mathrm{T}$ cells. ${ }^{38}$ Preclinical data using breast cancer models suggest that treatment with radiation and PD-1 inhibition can induce endogenous antigen-specific immune responses. ${ }^{39}$ In orthotopic brain mouse models, radiation in combination with PD-1 inhibition resulted in increased survival compared with either treatment alone. ${ }^{40} \mathrm{~A}$ unique phenomena seen with radiation and immunotherapy is the abscopal effect, wherein tumor regression in other metastatic sites can be observed after treatment with radiation; this has been reported in a patient with melanoma treated with radiation and ipilimumab. ${ }^{41}$ 
The combination of radiation and immune checkpoint blockade has been investigated in a small pilot study of 26 patients with breast cancer and brain metastases. Six patients with HER2-positive disease were treated with trastuzumab, tremelimumab, and whole-brain radiation. One patient experienced a non-central nervous system (CNS) partial response and another experienced SD; both were durable responses lasting at least 6 months. In 20 patients with HER-negative disease, 2 experienced non-CNS SD. ${ }^{42}$ Another small pilot study investigated the combination of pembrolizumab with radiation $(3,000$ Gy in 5 fractions) in 17 patients with metastatic TNBC. Only 9 patients were evaluable for response due to rapid disease progression; however, $3(33 \%)$ had a partial response and $1(11 \%)$ had SD, and responses outside the radiation field were observed in $3(33 \%) .{ }^{43}$ Although these are small studies and the response rates are modest, this provides clear evidence of durable abscopal effects. Furthermore, these studies demonstrate the importance of patient selection; many of the patients were inevaluable due to rapidly progressive disease. A number of ongoing trials are investigating radiation in combination with immunotherapy (ClinicalTrial.gov identifiers: NCT02303366, NCT03051672, NCT03366844).

\section{Epigenetic Approaches}

Posttranslational modification can regulate genes involved in immune recognition, opening the possibility of "priming" tumors for immunotherapy. 5-azacitidine is a pyrimidine nucleoside analog that inhibits DNA methyltransferase, resulting in hypomethylation in a number of genes, and can thus regulate gene expression. In vitro studies in breast cancer cell lines have demonstrated that treatment with 5-azacitidine results in enrichment of genes involved in immunomodulatory pathways, including interferon signaling, antigen processing and presentation, and cytokine expression. ${ }^{44}$ Preclinical studies have also shown that azacitidine may upregulate PD-L1. ${ }^{45}$ Histone deacylase inhibitors have also been found to have immunomodulatory effects. The ENCORE 301 study investigating exemestane \pm entinostat found that treatment with entinostat led to decreases in granulocytic and monocytic myeloidderived stem cells (which inhibit T-cell proliferation and Th1 cytokine secretion) and increases in HLA-DR expression on CD14-positive monocytes. ${ }^{46}$
Studies combining epigenetic therapy with immunotherapy are ongoing (ClinicalTrials.gov identifiers: NCT02453620, NCT02708680).

\section{Other Targeted Combinations}

The concept of priming tumors for immunotherapy may also be applied to numerous other classes of agents. Cyclin-dependent kinases 4 and 6 (CDK4/6) inhibitors interrupt the cell cycle and have been found to have clinical benefit in patients with metastatic ER-positive disease. ${ }^{47,48}$ Furthermore, CDK4/6 inhibitors have been shown to recruit TILs in the neoadjuvant setting, and thus may be an important primer for immunotherapies. ${ }^{49}$ Studies investigating the role of CDK4/6 inhibitors with immunotherapy are ongoing (ClinicalTrials.gov identifier: NCT03147287).

PARP inhibitors have demonstrated improved survival in patients with metastatic HER2-negative breast cancers and germline BRCA mutations, and have now been FDA-approved. ${ }^{50}$ BRCA mutations can result in DNA repair defects through homologydirected repair (HDR) deficiency. Abnormalities in other DNA repair mechanisms, such as mismatch repair (MMR) deficiency, can be associated with very high mutational burden, rendering tumors sensitive to PD-1 inhibition..$^{51}$ Furthermore, preclinical studies suggest that PARP inhibitors can upregulate PDL1 in breast cancer models, which may make them a suitable combination agent for immunotherapy. ${ }^{52}$ A recent phase I study in women's cancers investigating the PD-L1 inhibitor durvalumab in combination with the PARP inhibitor olaparib found an ORR of $17 \%$; however, many patients experienced $\mathrm{SD} \geq 4$ months, and the disease control rate (DCR) was $83 \% .{ }^{53} \mathrm{~A}$ randomized phase II study is underway to investigate olaparib \pm atezolizumab in metastatic TNBC that is HDR-deficient (ClinicalTrials.gov identifier: NCT02849496). The TOPACIO/KEYNOTE-162 study was a single-arm phase II study that found patients with metastatic TNBC treated with niraparib and pembrolizumab had an ORR of $28 \%(n=46)$, and durable responses were observed irrespective of PD-L1 or BRCA status. ${ }^{54}$

\section{Immune-Related Biomarkers}

\section{Tumor Infiltrating Lymphocytes}

TILs are associated with response to chemotherapy and prognosis in breast cancer. In the neoadjuvant 
setting, numerous studies have shown a correlation between stromal TILs and pCR, particularly in TNBC and HER2-positive breast cancer. ${ }^{55}$ In the adjuvant setting, increased stromal TIL levels at diagnosis in TNBC are associated with better outcomes after anthracycline-based chemotherapy. ${ }^{56}$ Similarly, stromal TILs have been associated with better outcomes after trastuzumab-based therapy in patients with HER2-positive breast cancer in the adjuvant setting. ${ }^{57}$ Stromal TIL level at baseline may also help identify responders to immunotherapy. In a study evaluating atezolizumab in metastatic breast cancer, tumors with $>10 \%$ TILs had a higher DCR than those with TILs $\leq 10 \%$ ( $19 \%$ vs $7 \%$, respectively); furthermore, median OS was 12.6 versus 6.6 months, respectively $(P=.0028) .{ }^{58}$ Stromal TILs were also examined in the KEYNOTE-086 study, which found that median levels were higher in cohort $B(17.5 \%$ vs $5 \%$ for cohort $A ; P<.001)$, which was the cohort that was treatment-naïve and had a better ORR. When both cohorts were combined, responders clearly had higher TIL levels than nonresponders $(37.5 \%$ vs $5 \%$, respectively; $\mathrm{P}<.001)$. In fact, TILs outperformed PD-L1 as a predictive marker, and in multivariate analysis, PD-L1 status did not add to the predictive model. ${ }^{59}$ Another critical observation made in this analysis was the distribution of TILs by organ site, where lymph nodes had high TILs and, notably, liver metastasis had low levels. Indeed, this may explain limited responses seen in patients with liver metastasis.

\section{Immunohistochemistry Approaches}

Like with other cancer histologies, PD-L1 overexpression by immunohistochemistry is associated with response in some studies; however, other studies have shown that patients with PD-L1-negative tumors may also exhibit responses, suggesting that PDL1 expression alone may not fully predict response to immunotherapy. ${ }^{10,12,60,61}$ The lack of standardization and variable cutoffs also hamper development of this biomarker. When combination CTLA-4 and PD-1 therapies are used, PD-L1 status is not associated with response in melanoma. ${ }^{28}$ Furthermore, when combined with chemotherapy in breast cancer, early initial studies suggest that PD-L1 status, although inconsistently defined across studies, may not be relevant. ${ }^{20,22}$ Although in the development of targeted therapies a biomarker of said target is sufficient to identify potential responders to a corresponding inhibitor (ie, HER2 and trastuzumab), immunecancer cell interactions are far more complex, and more comprehensive biomarkers may be required. One promising technology to capture this complex biology are multiplex immunohistochemical assays. Multiplex immunohistochemistry can evaluate several biomarkers concurrently on both the tumor cells and on the immune cells within the tumor microenvironment (ie, PD-L1, CTLA-4, LAG3, IDO), and are currently under investigation.

\section{Immunogenomics}

Immunogenomics is the genetic characterization of the immune system using DNA and RNA sequencing technologies and may be used to describe tumorimmune system interactions. Immune cells identify cancer cells by detecting antigens, termed neoantigens, expressed on cancer cells. Higher mutational burden may correlate with more diverse neoantigen landscapes and predict responses to immunotherapy in patients with melanoma and lung cancer. ${ }^{62,63} \mathrm{Sim}$ ilarly, patients with MMR-deficient cancers have a higher number of somatic mutations compared with those who are MMR-proficient, but furthermore, they have better responses to immune checkpoint blockade. ${ }^{64}$ Diverse neoantigen landscapes are more likely to stimulate neoantigen-specific $\mathrm{T}$ cells, which can be measured through T-cell receptor sequencing to quantify the clonality of the T-cell receptor repertoire. Preliminary studies in patients with melanoma and lung cancer suggest that patients with oligoclonal T-cell receptor repertoires are more likely to respond to immunotherapy. ${ }^{63,65}$ These immunogenomic biomarkers may, therefore, help identify patients with breast cancer more likely to benefit from immunotherapies. Clonal expansion of the T-cell receptor repertoire has also been associated with response to neoadjuvant therapy in early-stage breast cancer ${ }^{66}$ In the study investigating durvalumab and tremelimumab in metastatic breast cancer, investigators demonstrated that mutational and neoantigen burden was associated with immunosensitivity, and responders were found to have an oligoclonal expansion of the T-cell receptor repertoire, as well as upregulation of immune-related gene expression (CD8, granzyme A, perforin 1). ${ }^{67}$ The dynamic changes that can be assessed through immunogenomic markers may be important in identifying patients more likely to benefit from immunotherapy. 


\section{Conclusions and Future Directions}

Initial studies of immunotherapy in breast cancer have been useful in generating preliminary data to guide further development of these agents in breast cancer. We have established that a subset of breast tumors are immunogenic, and ORRs in TNBC are comparable with other malignancies for which singleagent ICIs have already been approved. As with other cancers, patients with breast cancer that responds to therapy tend to have durable clinical benefit and prolonged survival. Another crucial lesson learned from these studies is that clinical context matters, and this will help to inform patient selection for future studies. Findings from KEYNOTE-086 and the atezolizumab phase IA study suggest that a frontline approach results in higher responses rather than latter lines, pos- sibly because patients who are treatment-naïve have greater immune competency than those who have undergone multiple lines of chemotherapy. Gross disease burden, which may be estimated by LDH level or presence of visceral disease-in particular liver metastasis, which has been demonstrated to have low TILs-may also be a factor; patients in KEYNOTE-086 cohort A had higher LDH levels and visceral involvement and did worse than those in cohort B. Furthermore, time to response is longer than what is observed with chemotherapy, which may limit benefit in patients with rapid disease progression. These clinical factors present a dilemma in TNBC, which typically behaves aggressively and often manifests with significant visceral involvement. Thus, for most cases of TNBC, singleagent immunotherapy will likely be insufficient. Bio-

Table 2. Key Clinicopathologic Features and Response to Checkpoint Inhibitors

\begin{tabular}{|c|c|c|}
\hline $\begin{array}{l}\text { Clinicopathologic } \\
\text { Feature }\end{array}$ & Observation & Limitations and Alternative Strategies \\
\hline \multirow[t]{2}{*}{ Line of therapy } & $\begin{array}{l}\text { Highest response rates seen in the frontline setting in } \\
\text { metastatic TNBC }\end{array}$ & \multirow[t]{2}{*}{$\begin{array}{l}\text { Integration with biological factors to provide rationale for this } \\
\text { clinical observation }\end{array}$} \\
\hline & $\begin{array}{l}\text { Emerging data in early-stage setting in combination } \\
\text { with chemotherapy demonstrates highest response } \\
\text { rates to date }\end{array}$ & \\
\hline \multirow[t]{2}{*}{ TILs } & \multirow{2}{*}{$\begin{array}{l}\text { Higher level of stromal TILs in tumor microenvironment } \\
\text { associated with higher response rates in metastatic } \\
\text { breast cancer }\end{array}$} & Emerging data suggest that TILs may vary by metastatic site \\
\hline & & $\begin{array}{l}\text { Characterization of T cells in tumor microenvironment likely } \\
\text { important (ie, CD8+ by IHC, TCR sequencing) }\end{array}$ \\
\hline \multirow[t]{3}{*}{ PD-L1 status } & \multirow[t]{3}{*}{$\begin{array}{l}\text { Appears to correlate with higher response rates in } \\
\text { some studies }\end{array}$} & $\begin{array}{l}\text { Not consistently associated with response to checkpoint } \\
\text { monotherapy }\end{array}$ \\
\hline & & $\begin{array}{l}\text { Does not correlate at all with response to checkpoint inhibitor } \\
\text { chemotherapy combinations }\end{array}$ \\
\hline & & $\begin{array}{l}\text { Lack of uniform assessment of PD-L1 status (variable cut points } \\
\text { and antibodies used to determine positivity in literature) }\end{array}$ \\
\hline \multirow[t]{3}{*}{$\begin{array}{l}\text { Disease burden/ } \\
\text { visceral involvement }\end{array}$} & $\begin{array}{l}\text { Patients with TNBC and hepatic metastases have low } \\
\text { response rates to anti-PD-1/PD-L1 monotherapy }\end{array}$ & $\begin{array}{l}\text { Combination strategies may be able to convert "cold" to } \\
\text { "hot" disease }\end{array}$ \\
\hline & $\begin{array}{l}\text { Correlative studies suggest lower level of TILs present } \\
\text { in hepatic metastases }\end{array}$ & \multirow[t]{2}{*}{ Optimal combination strategies remain to be determined } \\
\hline & Elevated LDH associated with poor response to therapy & \\
\hline \multirow[t]{2}{*}{ Time to response } & \multirow[t]{2}{*}{$\begin{array}{l}\text { Time to response for single-agent checkpoint inhibitors } \\
\text { is typically }>8 \text { weeks }\end{array}$} & $\begin{array}{l}\text { Monotherapy of limited value in those with rapidly } \\
\text { progressive disease }\end{array}$ \\
\hline & & Chemotherapy combinations may improve time to response \\
\hline \multirow[t]{3}{*}{$\begin{array}{l}\text { Rational drug } \\
\text { combination }\end{array}$} & $\begin{array}{l}\text { A number of checkpoint inhibitor combination } \\
\text { strategies are being explored }\end{array}$ & $\begin{array}{l}\text { Most combination trials reported to date are single-arm } \\
\text { studies, limiting assessment of synergy }\end{array}$ \\
\hline & $\begin{array}{l}\text { Combinations with chemotherapy and other targeted } \\
\text { therapies appear well tolerated }\end{array}$ & $\begin{array}{l}\text { Overwhelming number of combinations being explored, need } \\
\text { to justify in the context of robust scientific/preclinical data }\end{array}$ \\
\hline & $\begin{array}{l}\text { Data from neoadjuvant setting suggest synergy with } \\
\text { chemotherapy }\end{array}$ & $\begin{array}{l}\text { Need to identify biomarkers to determine which tumor is most } \\
\text { likely to respond to any given treatment strategy }\end{array}$ \\
\hline \multirow[t]{3}{*}{ Breast cancer subtype } & \multirow{2}{*}{$\begin{array}{l}\text { HER2-positive and TNBC appear to be the most } \\
\text { immunogenically "hot" tumors (have highest level of } \\
\text { TILs) }\end{array}$} & Response to monotherapy remains low across all subtypes \\
\hline & & \multirow[t]{2}{*}{$\begin{array}{l}\text { Most breast cancers are "cold" and combination approaches } \\
\text { to enhance immunogenicity are needed }\end{array}$} \\
\hline & $\begin{array}{l}\text { Most ER-positive breast cancer appears to be } \\
\text { immunogenically "cold" }\end{array}$ & \\
\hline
\end{tabular}

Abbreviations: ER, estrogen receptor; IHC, immunohistochemistry; LDH, lactate dehydrogenase; TCR, T-cell receptor; TILs, tumor-infiltrating lymphocytes; TNBC, triple-negative breast cancer. 
Immune Checkpoint Inhibitors in Breast Cancer

logically rational combination strategies informed by preclinical investigations are critical for efficient and successful clinical development. Because single-agent responses are low, patient identification is crucial and predictive biomarkers are urgently needed. PDL1 status may not be sufficient, thus a better understanding of the biological interaction between cancer and the immune system and its microenvironment is required to develop comprehensive biomarkers that fully capture phenotypes most likely to benefit from immunotherapy. Given that most breast cancers are immunologically silent, or "cold," combination strategies to turn these tumors "hot" will be particularly crucial. Rethinking breast cancer classification according to immunophenotype may also be necessary to distinguish patients who may benefit from singleagent therapy from those who require combination strategies. Table 2 reviews some key clinicopathologic features that appear to impact response to ICIs.

Although the field of immunotherapy is relatively nascent in breast cancer, clinical activity has been established. Breast cancer has been the prototypic cancer histology, in which translational research has led to powerful targeted therapies and better patient outcomes, with almost a quarter million new diagnoses and 40,000 breast cancer-related deaths a year; however, much remains to be accomplished. Because of its prevalence, the opportunity to perform neoadjuvant studies due to ease of tissue acquisition, and its immunophenotypic heterogeneity, breast cancer is an ideal malignancy in which to study immunotherapy strategies. This may not only result in better outcomes for patients with breast cancer but also lead the way for translational immunotherapy development in other cancer types.

\section{References}

1. Dunn GP, Bruce AT, Ikeda $H$, et al. Cancer immunoediting: from immunosurveillance to tumor escape. Nat Immunol 2002;3:991-998.

2. Hodi FS, O'Day SJ, McDermott DF, et al. Improved survival with ipilimumab in patients with metastatic melanoma. N Engl J Med 2010;363:711-723.

3. Topalian SL, Hodi FS, Brahmer JR, et al. Safety, activity, and immune correlates of anti-PD-1 antibody in cancer. N Engl J Med 2012;366:24432454 .

4. Brahmer JR, Tykodi SS, Chow LQ, et al. Safety and activity of anti-PD-L1 antibody in patients with advanced cancer. N Engl J Med 2012;366:24552465.

5. Robert C, Schachter J, Long GV, et al. Pembrolizumab versus ipilimumab in advanced melanoma. N Engl J Med 2015;372:2521-2532.

6. Robert C, Long GV, Brady B, et al. Nivolumab in previously untreated melanoma without BRAF mutation. N Engl J Med 2015;372:320-330.

7. Brahmer J, Reckamp KL, Baas $P$, et al. Nivolumab versus docetaxel in advanced squamous-cell non-small-cell lung cancer. N Engl J Med 2015;373:123-135.

8. Perou CM, Sorlie T, Eisen MB, et al. Molecular portraits of human breast tumours. Nature 2000;406:747-752.

9. Tobin NP, Harrell JC, Lovrot J, et al. The molecular subtype and tumor characteristics of breast cancer metastases significantly influence patient post-relapse survival. Ann Oncol 2015;26:81-88.

10. Nanda R, Chow LQ, Dees EC, et al. Pembrolizumab in patients with advanced triple-negative breast cancer: phase Ib KEYNOTE-012 study. J Clin Oncol 2016;34:2460-2467.

11. Adams S, Loi S, Toppmeyer D, et al. Phase 2 study of pembrolizumab as first-line therapy for PD-L1-positive metastatic triple-negative breast cancer (mTNBC): preliminary data from KEYNOTE-086 cohort B [abstract]. J Clin Oncol 2017;35(Suppl):Abstract 1088.

12. Adams S, Schmid P, Rugo HS, et al. Phase 2 study of pembrolizumab (pembro) monotherapy for previously treated metastatic triple-negative breast cancer (mTNBC): KEYNOTE-086 cohort A [abstract]. J Clin Oncol 2017;35(Suppl):Abstract 1008.

13. Schmid P, Cruz C, Braiteh FS, et al. Atezolizumab in metastatic TNBC (mTNBC): long-term clinical outcomes and biomarker analyses [abstract]. Cancer Res 2017;77(13 Suppl):Abstract 2986.

14. Dirix LY, Takacs I, Jerusalem G, et al. Avelumab, an anti-PD-L1 antibody, in patients with locally advanced or metastatic breast cancer: a phase $1 \mathrm{~b}$ JAVELIN Solid Tumor study. Breast Cancer Res Treat 2018;167:671-686.

15. Vonderheide RH, LoRusso PM, Khalil M, et al. Tremelimumab in combination with exemestane in patients with advanced breast cancer and treatment-associated modulation of inducible costimulator expression on patient T cells. Clin Cancer Res 2010;16:3485-3494.

16. Dirix LY, Takacs I, Nikolinakos P, et al. Avelumab (MSB0010718C), an anti-PD-L1 antibody, in patients with locally advanced or metastatic breast cancer: a phase Ib JAVELIN solid tumor trial [abstract]. Presented at the 38th annual CTRC-AACR San Antonio Breast Cancer Symposium; December 8-12, 2015; San Antonio, Texas. Abstract S1-04.

17. Rugo HS, Delord JP, Im SA, et al. Preliminary efficacy and safety of pembrolizumab (MK-3475) in patients with PD-L1-positive estrogen receptor-positive/HER2-negative advanced breast cancer enrolled in KEYNOTE-028 [abstract]. Presented at the 38th Annual CTRC-AACR San Antonio Breast Cancer Symposium; December 8-12, 2015; San Antonio, Texas. Abstract S5-07.

18. Loi S, Giobbie-Hurder S, Gombos S, et al. Phase Ib/II study evaluating safety and efficacy of pembrolizumab and trastuzumab in patients with trastuzumab-resistant HER2-positive advanced breast cancer: results from the PANACEA study (IBCSG 45-13/BIG 4-13/KEYNOTE-014) [abstract]. Presented at the 2017 San Antonio Breast Cancer Symposium; December 5-9, 2017; San Antonio, Texas. Abstract GS2-06.

19. Zitvogel L, Galluzzi L, Smyth MJ, Kroemer G. Mechanism of action of conventional and targeted anticancer therapies: reinstating immunosurveillance. Immunity 2013;39:74-88.

20. Adams S, Diamond JR, Hamilton EP, et al. Phase Ib trial of atezolizumab in combination with nab-paclitaxel in patients with metastatic triplenegative breast cancer (mTNBC) [abstract]. J Clin Oncol 2016;34(15 Suppl):Abstract 1009.

21. Emens LA, Adams S, Loi S, et al. IMpassion130: a phase III randomized trial of atezolizumab with nab-paclitaxel for first-line treatment of patients with metastatic triple-negative breast cancer (mTNBC) [abstract]. J Clin Oncol 2016;34(15 Suppl):Abstract TPS1104-TPS

22. Tolaney SM, Kalinsky K, Kaklamani V, et al. Phase $1 \mathrm{~b} / 2$ study to evaluate eribulin mesylate in combination with pembrolizumab in patients with metastatic triple-negative breast cancer [abstract]. Presented at the 2017 San Antonio Breast Cancer Symposium; December 6-10, 2016; San Antonio, Texas. Abstract PD6-13.

23. Page DB, Kim IK, Sanchez K, et al. Safety and efficacy of pembrolizumab (pembro) plus capecitabine (cape) in metastatic triple negative breast cancer (mTNBC) [abstract]. J Clin Oncol 2018;36(Suppl):Abstract 1033.

24. Kok M, Voorwerk L, Horlings $H$, et al. Adaptive phase II randomized trial of nivolumab after induction treatment in triple negative breast cancer (TONIC trial): final response data stage I and first translational data [abstract]. J Clin Oncol 2018;36(Suppl):Abstract 1012.

25. Nanda R, Liu MC, Yau C, et al. Pembrolizumab plus standard neoadjuvant therapy for high-risk breast cancer (BC): results from I-SPY 2 [abstract]. J Clin Oncol 2017;35(Suppl):Abstract 506. 
26. LoiblS, Untch M, BurchardiN, etal. A randomized phase II neoadjuvant study (GeparNuevo) to investigate the addition of durvalumab, a PD-L1 antibody, to a taxane-anthracycline containing chemotherapy in triple negative breast cancer (TNBC) [abstract]. J Clin Oncol 2017;35(Suppl):Abstract 3062.

27. Curran MA, Montalvo W, Yagita H, Allison JP. PD-1 and CTLA-4 combination blockade expands infiltrating $\mathrm{T}$ cells and reduces regulatory $\mathrm{T}$ and myeloid cells within B16 melanoma tumors. Proc Natl Acad Sci U S A 2010;107:4275-4280

28. Larkin J, Chiarion-Sileni V, Gonzalez R, et al. Combined nivolumab and ipilimumab or monotherapy in untreated melanoma. N Engl J Med 2015;373:23-34

29. Santa-Maria CA, Kato T, Park JH, et al. A pilot study of durvalumab and tremelimumab and immunogenomic dynamics in metastatic breast cancer. Oncotarget 2018;9:18985-18996.

30. Zakharia $Y, M c W i l l i a m s ~ R$, Shaheen $M$, et al. Interim analysis of the phase 2 clinical trial of the IDO pathway inhibitor indoximod in combination with pembrolizumab for patients with advanced melanoma. Presented at the AACR Annual Meeting 2017; April 1-5, 2017; Washington, DC.

31. Ernst B, Anderson KS. Immunotherapy for the treatment of breast cancer. Curr Oncol Rep 2015;17:5.

32. Mittendorf EA, Clifton GT, Holmes JP, et al. Final report of the phase I/II clinical trial of the E75 (nelipepimut-S) vaccine with booster inoculations to prevent disease recurrence in high-risk breast cancer patients. Ann Oncol 2014;25:1735-1742.

33. Chen G, Gupta R, Petrik S, et al. A feasibility study of cyclophosphamide, trastuzumab, and an allogeneic GM-CSF-secreting breast tumor vaccine for HER2+ metastatic breast cancer. Cancer Immunol Res 2014;2:949-961.

34. Schumacher TN, Schreiber RD. Neoantigens in cancer immunotherapy. Science 2015;348:69-74.

35. Sabel MS, Nehs MA, Su G, et al. Immunologic response to cryoablation of breast cancer. Breast Cancer Res Treat 2005;90:97-104.

36. McArthur HL, Diab A, Page DB, et al. A pilot study of preoperative single-dose ipilimumab and/or cryoablation in women with early-stage breast cancer with comprehensive immune profiling. Clin Cancer Res 2016;22:5729-5737.

37. Page DB, Yuan J, Redmond D, et al. Deep sequencing of T-cell receptor DNA as a biomarker of clonally expanded TILs in breast cancer after immunotherapy. Cancer Immunol Res 2016;4:835-844.

38. Weichselbaum RR, Liang H, Deng L, Fu YX. Radiotherapy and immunotherapy: a beneficial liaison? Nat Rev Clin Oncol 2017;14:365-379.

39. Sharabi AB, Tran PT, Lim M, et al. Stereotactic radiation therapy combined with immunotherapy: augmenting the role of radiation in local and systemic treatment. Oncology (Williston Park) 2015;29:331-340.

40. Zeng J, See AP, Phallen J, et al. Anti-PD-1 blockade and stereotactic radiation produce long-term survival in mice with intracranial gliomas. Int J Radiat Oncol Biol Phys 2013;86:343-349.

41. Postow MA, Callahan MK, Barker CA, et al. Immunologic correlates of the abscopal effect in a patient with melanoma. N Engl J Med 2012;366:925931.

42. McArthur HL, Beal K, Halpenny D, et al. CTLA4 blockade with HER2directed therapy $(\mathrm{H})$ yields clinical benefit in women undergoing radiation therapy (RT) for HER2-positive (HER2+) breast cancer brain metastases (BCBM) [abstract]. Presented at the AACR Annual Meeting 2017; April 1-5, 2017; Washington, DC. Abstract 4705.

43. McArthur HL, Barker CA, Gucalp A, et al. A single-arm, phase II study assessing the efficacy of pembrolizumab (pembro) plus radiotherapy (RT) in metastatic triple negative breast cancer (mTNBC) [abstract]. J Clin Oncol 2018;36(Suppl):Abstract 14.

44. Chawla A, Philips AV, Alatrash G, Mittendorf E. Immune checkpoints: a therapeutic target in triple negative breast cancer. Oncoimmunology 2014;3:e28325.

45. Wrangle J, Wang W, Koch A, et al. Alterations of immune response of nonsmall cell lung cancer with azacytidine. Oncotarget 2013;4:2067-2079.

46. Tomita Y, Lee MJ, Lee $\mathrm{S}$, et al. The interplay of epigenetic therapy and immunity in locally recurrent or metastatic estrogen receptor-positive breast cancer: correlative analysis of ENCORE 301, a randomized, placebo-controlled phase II trial of exemestane with or without entinostat. Oncoimmunology 2016;5:e1219008.

47. Finn RS, Crown JP, Lang I, et al. The cyclin-dependent kinase $4 / 6$ inhibitor palbociclib in combination with letrozole versus letrozole alone as first-line treatment of oestrogen receptor-positive, HER2-negative, advanced breast cancer (PALOMA-1/TRIO-18): a randomised phase 2 study. Lancet Oncol 2015;16:25-35.
48. Cristofanilli M, Turner NC, Bondarenko I, et al. Fulvestrant plus palbociclib versus fulvestrant plus placebo for treatment of hormone-receptor-positive, HER2-negative metastatic breast cancer that progressed on previous endocrine therapy (PALOMA-3): final analysis of the multicentre, doubleblind, phase 3 randomised controlled trial. Lancet Oncol 2016;17:425-439.

49. Martin M, Hurvitz SA, Chan D, et al. Final results of NeoMONARCH: a phase 2 neoadjuvant study of abemaciclib in postmenopausal women with hormone receptor positive (HR+), HER2 negative breast cancer (BC) [abstract]. Presented at the 2016 San Antonio Breast Cancer Symposium; December 6-10, 2016; San Antonio, Texas.

50. Robson ME, Im SA, Senkus E, et al. OlympiAD: phase III trial of olaparib monotherapy versus chemotherapy for patients (pts) with HER2-negative metastatic breast cancer ( $\mathrm{mBC})$ and a germline BRCA mutation (gBRCAm) [abstract]. J Clin Oncol 2017;35(Suppl):Abstract LBA4-LBA.

51. Le DT, Uram JN, Wang H, et al. PD-1 blockade in tumors with mismatchrepair deficiency. N Engl J Med 2015;372:2509-2520.

52. Jiao S, Xia W, Yamaguchi $H$, et al. PARP inhibitor upregulates PD-L1 expression and enhances cancer-associated immunosuppression. Clin Cancer Res 2017;23:3711-3720.

53. Lee JM, Cimino-Mathews A, Peer CJ, et al. Safety and clinical activity of the programmed death-ligand 1 inhibitor durvalumab in combination with poly (ADP-ribose) polymerase inhibitor olaparib or vascular endothelial growth factor receptor 1-3 inhibitor cediranib in women's cancers: a dose-escalation, phase I study. J Clin Oncol 2017;35:2193-2202.

54. Vinayak S, Tolaney SM, Schwartzberg LS, et al. TOPACIO/KEYNOTE-162: niraparib + pembrolizumab in patients (pts) with metastatic triple-negative breast cancer (TNBC), a phase 2 trial [abstract]. J Clin Oncol 2018;36(15 Suppl):Abstract 1011 .

55. Denkert C, Loibl S, Noske A, et al. Tumor-associated lymphocytes as an independent predictor of response to neoadjuvant chemotherapy in breast cancer. J Clin Oncol 2010;28:105-113.

56. Loi S, Sirtaine N, Piette F, et al. Prognostic and predictive value of tumorinfiltrating lymphocytes in a phase III randomized adjuvant breast cancer trial in node-positive breast cancer comparing the addition of docetaxel to doxorubicin with doxorubicin-based chemotherapy: BIG 02-98. J Clin Oncol 2013;31:860-867.

57. Loi S, Michiels S, Salgado R, et al. Tumor infiltrating lymphocytes are prognostic in triple negative breast cancer and predictive for trastuzumab benefit in early breast cancer: results from the FinHER trial. Ann Oncol 2014;25:1544-1550

58. Schmid P, Cruz C, Braiteh FS, et al. Atezolizumab in metastatic TNBC (mTNBC): long-term clinical outcomes and biomarker analyses [abstract] Presented at the AACR Annual Meeting 2017; April 1-5, 2017 Washington, DC. Abstract 2986.

59. Loi S, Adams S, Schmid P, et al. Relationship between tumor infiltrating lymphocyte levels and response to pembrolizumab in metastatic triplenegative breast cancer: results from KEYNOTE-086 [abstract]. Ann Oncol 2017;28(Suppl 5):Abstract LBA13.

60. Callahan MK. Understanding the biology behind responses to immunotherapy. Presented at the 2014 ASCO Annual Meeting; May 30 June 4, 2014; Chicago, Illinois.

61. Brahmer JR, Drake CG, Wollner I, et al. Phase I study of single-agent antiprogrammed death-1 (MDX-1106) in refractory solid tumors: safety, clinical activity, pharmacodynamics, and immunologic correlates. J Clin Oncol 2010;28:3167-3175

62. Rizvi NA, Hellmann MD, Snyder A, et al. Cancer immunology. Mutational landscape determines sensitivity to PD-1 blockade in non-small cell lung cancer. Science 2015;348:124-128.

63. Snyder A, Makarov V, Merghoub T, et al. Genetic basis for clinical response to CTLA-4 blockade in melanoma. N Engl J Med 2014;371:2189-2199.

64. Salgado R, Denkert C, Demaria $S$, et al. The evaluation of tumor-infiltrating lymphocytes (TILs) in breast cancer: recommendations by an International TILs Working Group 2014. Ann Oncol 2015;26:259-271.

65. Tumeh PC, Harview CL, Yearley JH, et al. PD-1 blockade induces responses by inhibiting adaptive immune resistance. Nature 2014;515:568-571.

66. Park JH, Jang M, Tarhan YE, et al. Clonal expansion of antitumor T cells in breast cancer correlates with response to neoadjuvant chemotherapy. Int J Oncol 2016;49:471-478.

67. Santa-Maria CA, Kato T, Park JH, et al. Durvalumab and tremelimumab in metastatic breast cancer (MBC): immunotherapy and immunopharmacogenomic dynamics [abstract]. J Clin Oncol 2017;35(Suppl):Abstract 3052. 